\title{
A Review of Detection and Reduction of Noise in Degraded Images by Efficient Noise Detection Algorithm
}

\author{
Gayatri P. Bhelke \\ Sipna college of engineering \\ and technology, Amravati \\ Akola, India
}

\author{
V. S. Gulhane \\ Sipna college of engineering \\ and technology, Amravati \\ Amravati , India
}

\author{
N. D. Shelokar \\ Sipna college of engineering \\ and technology, Amravati \\ Amravati , India
}

\begin{abstract}
Noise is unwanted information present in image that can harm the quality of image. A novel noise detection and reduction technique is proposed in this paper. Technique is used to detect and reduce the noise in the degraded images. The main approach of this proposed technique is detecting and reducing different types of noise present in degraded images. Paper introduces a two step process where first step is used to detect the type of noise which is present in degraded image and second step handles the errors and compensate for various noise sources common in multimedia application, such as Salt and Pepper, Gaussian and speckle noise.
\end{abstract}

Keywords: Image Restoration, Degraded Images, Denoising, Noise Detection, Salt and pepper Noise, Gaussian .

\section{INTRODUCTION}

Noise is unwanted information which is present in the images, which affect the quality of images. Noise can be unavoidable in communication networks, and its presence can have terrible effects upon the data being sent [1]. Image Processing is a technique that improves the quality of raw Images capture in normal day-to-day life for many applications. Images captured by digital cameras could be affected by noise due to random variations of pixel elements in the camera sensors. There are various types of image noise present in the image such as Gaussian noise, salt and pepper noise, random valued impulse noise, speckle noise, Uniform noise [4].

\section{- $\quad$ Salt And Pepper Noise}

Salt and Pepper noise is also known as Impulse Noise. This noise can be caused by sharp \& sudden disturbances in the image signal. It represents itself is randomly occurring white or black (or both) dots over image.

\section{- Gaussian Noise}

Gaussian Noise is caused by random fluctuations in the signal. Its modeled by random values added to an image

\section{- $\quad$ Speckle Noise}

Speckle noise can be modeled by random values multiplied by pixel values of an image.

\section{- Uniform Noise}

Uniform noise is also known as quantization noise. It is caused by quantizing the pixels of a sensed image to a number of discrete levels. It has an approximately uniform distribution

In this work, we will present a new, faster and more efficient noise detection and reduction method for degraded images. The algorithm which is used to detect the presence of noise and to remove it, should be theoretically, and computationally as simple as possible. A well-defined process of detecting and reducing certain types of noise in transmitted images would have to be a somewhat crude for speed. The main aim of noise reduction is to smother the noise, and also probably to safeguard the harpness of edge and feature information [23].
Here, we have discussed a new efficient noise detection algorithm that will be able to allow the receiver to know what type of filtering method should be applied for the type of noise detected in given image.

\section{RELATED WORK}

Many of the current papers dealing with noise in communication networks which propose a two-stage method of impulse noise reduction where in the first stage noise is detected and in the second it compensated for a filtering technique.

As per Ming Yan's paper [10] when the noise level is not high, adaptive center-weighted median filter (ACWMF) is appropriate method for removing random-valued impulse noise. Paper presents a general algorithm for blind image inpainting and removing impulse noise by iteratively restoring the image and identifying the damaged pixels.

In H. Hosseini, F. Marvasti's [5] paper GFN(General Fixed-Valued Impulse Noise) model is used. GFN model required an Impulse Value Detector (IVD) to determine the noise values. In this received image is denoted as I and Image entrophy is defined as,

$$
\text { entropy }(I)=\sum_{i=0}^{255} \mathrm{pi} \log \mathrm{pi}
$$

where, pi is the probability of the grey-value $i$ and can be interpreted as the normalized histogram of the image. While the Gaussian noise does not affect the image entropy, the impulse noise significantly decreases it.

The impulse value detector, iteratively, detects and removes the impulse grey-values. If the corresponding pixels have the lowest correlation with their neighbors then the grey value is detected as an impulse. After each iteration, when the impulse value is removed, the image entropy increases. The process continues until the entropy becomes larger than the entropy threshold, thus it ensures that there are no more impulse values in the image and image restoration is done by using AIM filter. In this filter, the noisy pixels which are farther than their nearest uncorrupted pixel, will be modified in more iterations 
Qin Zhiyuan et.al 's[14] describe A Robust Adaptive Image Smoothing Algorithm in which analysis of some smoothing algorithms are given which include Edge Preserved filtering ,Adaptive medium filter, Robust smoothing filter and Gradient weighting filter. Robust adaptive algorithm combines multi-window templates, gradient weighting, constant gray output on non-pulse pixel and the improved adaptive smoothing algorithm.

In Addition to Smoothing algorithm, paper introduces the methods of enlarging windows and selecting sub template windows to remove salt and pepper noise with large space intensity. Because it uses a new algorithm by combining nonlinear filtering and linear filtering according to their respective adaptation to different noises.

In Deborah D. Duran-Herrmann et.al [1] paper two stage process is given in which first stage detect the type of noise and in second stage which type of filter is suitable for detected noise is given to eliminate the noise.

"Noise removal Algoritham for Image Corrupted by Additive Gaussian Noise"[20] describe two fundamental mathematical morphological operations, that are dilation and erosion. Dilation adds pixels to the boundaries of objects in an image, where as erosion removes pixels on object boundaries. Mathematical morphological operations are also useful in smoothing and sharpening. Paper presents noise removal algorithm for gray scale images corrupted by Gaussian noise.

\section{PROPOSED WORK}

Our given algorithm uses a two-stage process of determining three things which are given below,

\section{- $\quad$ Presence of noise \\ - Type of noise such as impulse noise or Gaussian \\ - The effective filtering method for removing noise}

Similar to [1], architecture includes Adaptive Noise Detector and Adaptive pixel Restorer

Following fig shows system architecture,

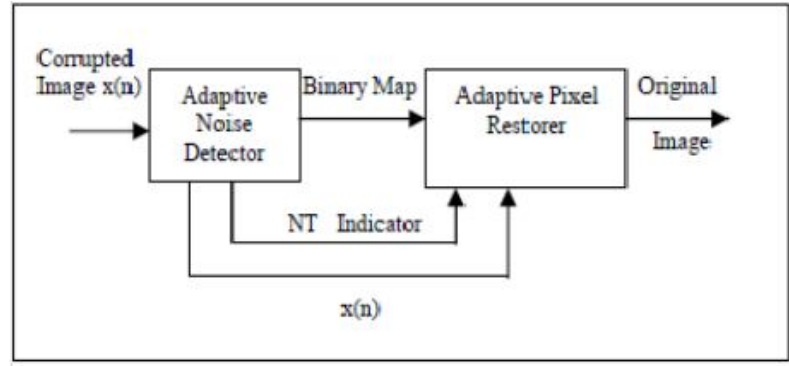

Figure. 1 . System Architecture

Following subsection describe Adaptive Noise Detector and Adaptive pixel Restorer.

\subsection{Adaptive Noise Detector}

The Adaptive Noise Detector is used to detect the type of noise such as Gaussian noise, salt and pepper and so on, if exists in the current image. Similar to [8] it follows the following steps,

Step 1: Obtain the image histogram $\mathrm{H}$ of the degraded image
Step2: Compute the vector $D$ which is the difference between adjacent locations in the histogram array $H$.

$D i=H i+1-H i$ for all $i=0,1 \ldots 255$

Step 3: Various boundary thresholds are set, according to the maxima values found in D. Depending on location of maxima values nature of the noise is detected, whether it be impulse, uniform, or Gaussian. Once the noise has been find out, the NT Indicator is set as an input to the second stage of the system. The corrupted pixels are then mapped to a binary matrix with the same dimensions as the image

\subsection{Adaptive pixel Restorer}

In second stage NT indicator is used as input which increases the processing speed. If the NT Indicator has not been set, the "corrupted image", $x(n)$, is allowed to pass and if the NT Indicator is set, then this sets one of the various noise flags. Again, similar to the second stage of [1], the Adaptive Pixel Restorer searches through the Binary Map for pixels whose value is "1". If the neighborhood vector of values is not set as null and instance is found, it searches noise flags that are set to determine what type of adaptive filtering is best for that instance which is courrpted. If the neighborhood vector of values in the vicinity of that particular pixel is null, the algorithm goes on to the next pixel value. This process is done repeatedly until the Binary Map is cleared of 1's.[1]

Following fig(a) shows the original image fig(b) shows image corrupted by salt and pepper noise with histogram , fig(c) shows image corrupted by the Gaussian noise with histogram

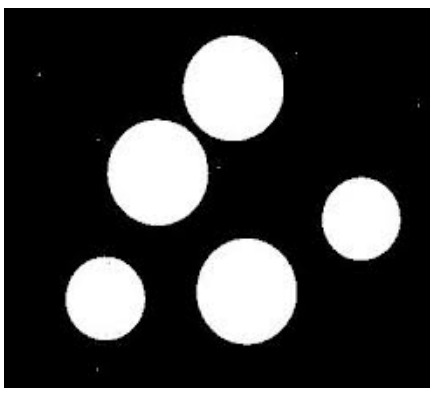

Figure(a) original Image

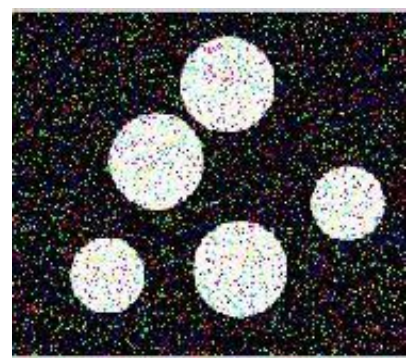




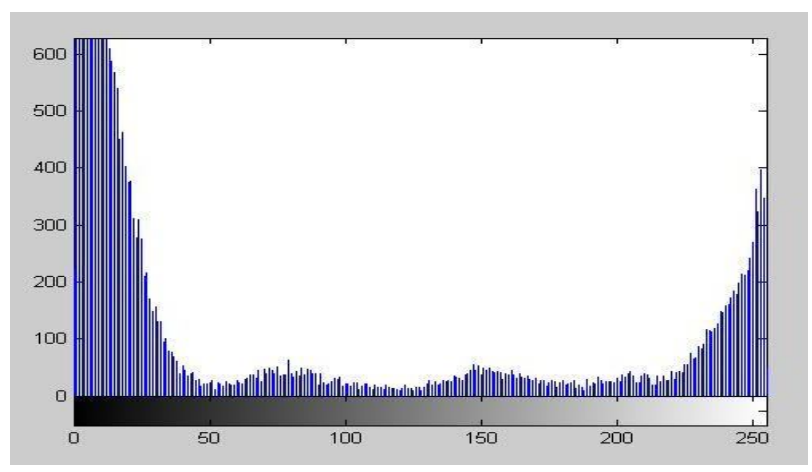

Figure (b) Image corrupted by Salt and pepper noise histogram of salt and pepper noise.
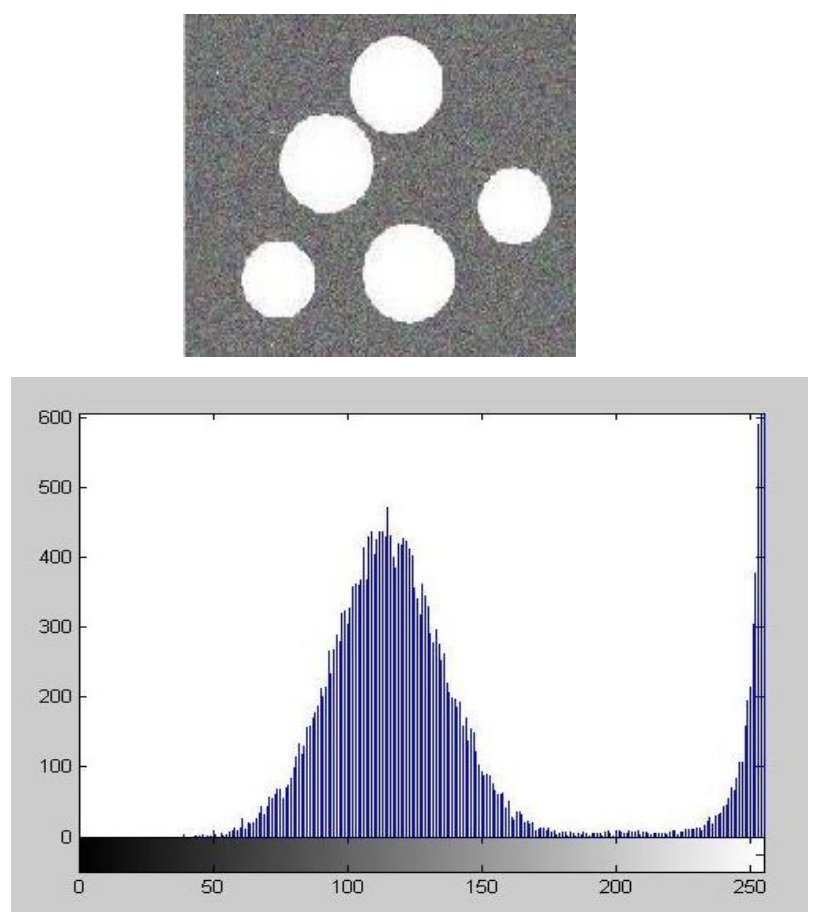

Figure (c). Image corrupted by Gaussian Noise with histogram

Objectives of this proposed work are summarized as follow

- Analysis of algorithm which used for detection of noise in corrupted images.

- Implementation of Adaptive/Novel Algorithm for detection and reduction of Noise in corrupted images.

- Implementation will be carried out in two step process,

$>$ Presence of noise

$>$ Type of noise

$>$ Effective filtering method for removing noise.
With the best level efforts, above one or more task may be tried to be implemented

\section{REFERENCES}

[1] [Deborah D. Duran-Herrmann et al 2012], Qilin Qi, and Yaoqing (Lamar) Yang "An Adaptive Algorithm for Corrupted Images" International Conference on Systems and Informatics (ICSAI 2012)

[2] [D. Ze-Feng et al 2007], Y. Zhou-Ping, and X. You-Lun "High Probability Impulse Noise- Removing Algorithm Based on Mathematical Morphology," IEEE Signal Processing Letters, Vol. 14, No. 1, January 2007, pp. 31-34.

[3] [Gouchol Pok et al 2003], Jyh-Chain Liu and A.S. Nair, "Selective removal of impulse noise based on homogeneity level information", IEEE Trans on image processing, vol.12, no.1, pp.85-92, January 2003.

[4] [Gurmeet kaur et al 2012] Rupindar Kaur "Image Denoising using Wavelet Transform and various filters", International journal of researcher in computer science Eissn 2249-8265 volume 2(2012)pp.15-21W.

[5] [H. Hosseini et al 2011], Senior Member, IEEE "Fast Impulse Noise Removal from highly corrupted image"

[6] [How-Lung Eng et al 2001], Kai-Kuang Ma," Noise adaptive soft switching median filter", IEEE Trans on Image process., vol.10, no.2, pp.242-251, Feb.2001.

[7] [Ho-Ming Lin et al 2007],Alan N Willson, "Median filters with adaptive Length", IEEE Trans on circuits and systems, vol.35,no.6, pp.675-690, June 1988.

[8] .[Indu S and Chaveli Ramesh 2007], "A Noise Fading Technique for Images Highly Corrupted with Impulse Noise" International Conference on Computing: Theory and Applications (ICCTA'07).

[9] [Luo, 2006], "Efficient Removal of Impulse Noise from Digital Images,"IEEE Transactions on Consumer Electronics, Vol. 52, No. 2, May 2006,pp. 523-527.

[10] [Ming yan 2011], "Restoration of Images Corrupted by Impulse Noise using Blind Inpainting and 10 Norm".

[11] [ Pei-Eng Ng et al 2006], Kai-Kuang Ma, "A switching median filter with BDND for extremely corrupted images", IEEE Trans on Image process., vol. 15, no.6, pp.1506-1516, June 2006.

[12] [Peter D. Wendt et al 1986], Edward J Coyle and Neal. C. Gallagher, "Some convergence properties of median filters", IEEE Transon circuits and systems, vol. cas-33, no.3, pp.276-286, March 1986

[13] [Ping et al 2007], W.; Junli, L.; Lu, D.; and Chen, G. "A Fast and Reliable Switching Median Filter for Highly Corrupted Images by Impulse Noise," IEEE Transactions, 2007, pp. 3427-3440.

[14] [Qin Zhiyuan a et al 2010] Zhang Weiqiang a, Zhang Zhanmu a, Wu Bing b, RuiJie a,ZhuBaoshan "A Robust Adaptive image smoothing algorithm"

[15] [R. Gonzales et al 2002] R. Woods "Digital Image Processing" Second Edition Prentice-Hall, Inc. 2002.

[16] [Raymond H Chan et al 2005], Chung-Wa Ho and Mila Nikolova, "Salt nd pepper noise removal by median type noise detectors and detail preserving regularization", 
IEEE Trans on Image Processing, vol.14, no.10, pp. 1479-1485, October 2005.

[17] [Reinhard Berstein 1987], "Adaptive nonlinear filters for simultaneous removal of different kinds of noise in images",IEEE Trans on circuits and systems, vol.Cas-34, no.11,pp.1275-1291, November 1987

[18] [S. Schulte, et al 2006] M. Nachtegael, V. De Witte, D. Van der Weken, E.E.Kerre, "A Fuzzy Impulse Noise Detection and Reduction Method,"IEEETransactions on Image Processing, Vol 15, Issue 5, May 2006, pp.1153 1162.

[19] [S. Schulte, M. Nachtegael et al 2006] V. De Witte, D. Van der Weken, E.E. Kerre, "Fuzzy Two-Step Filter for Impulse Noise Reduction From Color Images,"IEEE Transactions on Image Processing" Vol 15, No 11, November 2006, pp.3568-3579.

[20] [Shyam Lall et al 2009] Mahesh Chandra, Gopal Krishna Upadhya "Noise Removal Algoritham for Image Corrupted by Additive Gaussian Noise",International Journal of Recent trends in Engineering, vol 2,No.1,November 2009,pp199-206

[21] [T. Chen et al 2006] S. Huang, C. Chen, and Z. Lin, "Adaptive Working Window for Impulse Noise Reduction," International Conference on Intelligent Information Hiding and Multimedia Signal Processing, 2006.

[22] [Tao Chen et al 1999], Kai-Kuang Ma , Li- Hui- Chen, "Tristate median filter for image denoising", IEEE Trans on image processing, vol.8, no.12, pp.1834-1838, December 1999.

[23] [V. Saradhadevi et. al 2011], Dr.V.Sundaram, "An Enhanced Two-Stage Impulse Noise Removal Technique based on Fast ANFIS and Fuzzy Decision", IJCSI International Journal of Computer Science Issues, Vol. 8, Issue 5, No 1, September 2011.

[24] [W. Luo et al 2006 ] D. Dang, "An Efficient Method for the Removal of Impulse Noise," ICIP, 2006, pp. 26012604

[25] [W. Luo,2006] "An Efficient Detail-Preserving Approach for Removing Impulse Noise in Images," In IEEE Siganl Processing Letters, Vol 13, No 7, July 2006, pp. 413-416.

[26] [W. Luo,2006] "Efficient Removal of Impulse Noise from Digital Images,"IEEE Transactions on Consumer Electronics, Vol. 52, No. 2, May 2006,pp. 523-527

[27] [Xiaoyin Xu et al 2004], Eric L Miller, Dong bin Chen and Mansoor Sarhadi, "Adaptive two-pass rank order filter to remove impulse noise in highly corrupted images", IEEE Trans on image processing, vol.13, no.2, pp. 238-247, February 2004

[28] [Xu, H et al 2006], Xia, X.; Guo, L.; Chen, W.; and Huang, G. "Classification-based Weighted Filter for Image Corrupted by Impulse Noise," ICSP Proceedings, 2006. 